\title{
The catheter tip distensibility substantially influences the aspiration force of thrombectomy devices
}

Jiahui Li1, Oscar Castaño PhD2,3,4,5, Alejandro Tomasello MD6, Marta de Dios Lascuevas MD7, Pere Canals ${ }^{1}$, Elisabeth Engel PhD3,4,8, Marc Ribo MD PhD',9

1. Stroke Research Group, Vall d'Hebron Research Institute, Barcelona, Spain.

2. Electronics and Biomedical Engineering, University of Barcelona, Barcelona, Spain

3. Biomaterials for Regenerative Therapies, Institute for Bioengineering of Catalonia, The BarceIona Institute of Science and Technology (BIST), Barcelona, Spain.

4. CIBER en Bioingeniería, Biomateriales y Nanomedicina (CIBER-BBN), Madrid, Spain.

5. Institute of Nanoscience and Nanotechnology, Universitat de Barcelona (UB), 08028 Barcelona, Spain.

6. Department of Neuroradiology, Vall d'Hebron University Hospital, Barcelona, Spain.

7. Department of Neuroradiology, Bellvitge University Hospital, Hospitalet de Llobregat, Spain.

8. Materials Science and Metallurgy, EEBE, Technical University of Catalonia (UPC), Barcelona, Spain

9. Stroke Unit, Neurology Department, Vall d'Hebron University Hospital, Barcelona, Spain.

Corresponding author:

Marc Ribo, MD, PhD

Stroke Unit, Neurology Department, Vall d'Hebron University Hospital, Barcelona, Spain

Hospital Vall d'Hebron, Passeig de la Vall d'Hebron Barcelona 119-129, Spain

E-mail: marcriboj@hotmail.com

Cover title: Catheter tip distensibility and aspiration force

Keywords: Stroke treatment, endovascular treatment, aspiration force, thrombectomy, catheters Word count: 2286

Tables: 1 Figures: 3 


\section{SUMMARY}

Background A direct aspiration first pass thrombectomy (ADAPT) is a fast-growing technique for which a broad catalog of catheters that provide a wide range of aspiration forces can be used. We aim to characterize different catheters' aspiration performance on stiff clots in an in vitro vascular model. We hypothesize that labeled catheter inner diameter (labeled-ID) is not the only parameter that affects the aspiration force (asp-F) and that thrombus-catheter tip interaction and distensibility also play a major role.

Methods We designed an experimental setup consisting of a 3D-printed carotid artery immersed in a water deposit. We measured asp-F and distensibility of catheter tips when performing ADAPT on a stiff clot analog larger than catheter labeled-ID. Correlations between asp-F, catheter ID, and tip distensibility were statistically assessed.

Results Experimental asp-F and catheter labeled-ID were correlated $(r=0.9601, p<0.01)$. The relative difference between experimental and theoretical Asp- $F$ (obtained by the product of the tip's section area by the vacuum pressure) correlated with tip's distensibility $(r=0.9050, p<0.01)$, evidencing that ADAPT performance is highly influenced by catheter tip shape-adaptability to the clot and that the effective ID (eff-ID) may differ from labeled-ID specified by manufacturers. Eff-ID showed the highest correlation with experimental asp- $\mathrm{F}(\mathrm{r}=0.9944, \mathrm{p}<0.01)$, confirming that eff-ID rather than labeled-ID should be considered to better estimate the device efficiency.

Conclusions Catheter tip distensibility can induce a significant impact on ADAPT performance when retrieving a stiff clot larger than the device ID. Our findings might contribute to optimizing thrombectomy strategies and the design of novel aspiration catheters. 


\section{INTRODUCTION}

Mechanical thrombectomy is a minimally invasive procedure, rapidly growing worldwide, that provides the best clinical outcomes in ischemic strokes due to large vessel occlusion [1]-[3]. A direct aspiration first pass technique (ADAPT) is an emergent thrombectomy strategy that provides safe and rapid revascularization. Its effectivity has accelerated the development of endovascular devices [4].

The ADAPT approach generally consists of navigating a large-bore catheter through the vascular anatomy to reach the occlusion site, where the catheter tip is positioned close to or in direct contact with the proximal aspect of the thrombus. The catheter is then connected to a syringe or aspiration pump that generates vacuum pressure to either aspirate the thrombus through the catheter or engage it for subsequent retrieval.

The main approaches to evaluate the performance of aspiration devices in the literature are computational fluid dynamics analysis [5], [6], experimental flow rate measurements [7], [8], and studies related to aspiration force (asp-F) and vacuum pressure [9]-[11]. Technical evaluations by several authors suggest that the aspiration performance of commercially available devices is highly correlated to the labeled inner diameter (labeled-ID) of the catheter tip. Hence, the higher the catheter labeled-ID, the higher the asp-F [7]-[10], [12]. Recently, Yaeger et al. [10] studied the asp-F generated by an aspiration pump through several commercial catheters and found a correlation coefficient of 0.981 between catheter labeled-ID and the measured asp-F. Nevertheless, the same study reported that Stryker Cat 6 (labeled-ID $=0.06 \mathrm{in}$, asp-F $=130.57 \pm 4.12 \mathrm{mN}$ ) and Penumbra 5MAX (labeled-ID = $0.054 \mathrm{in}$; asp-F $=132.24 \pm 3.92 \mathrm{mN}$ ) delivered similar asp-F despite the significant difference in their labeled-ID, which suggests that catheter labeled-ID may not be the only parameter that determines asp-F.

The goal of this study is to determine whether catheter tip mechanical behavior impacts ADAPT performance. For this purpose, we define catheter tip distensibility as the capability of a device to enlarge its ID when it is interacting with a thrombus that cannot be completely ingested due to its size and stiffness. In this work, we evaluated the asp-F of several thrombectomy catheters, the percentage of catheter tip widening as a measure of tip distensibility, and the correlation between them.

\section{METHODS}

\section{Catheters}

The following thrombectomy catheters were evaluated: Penumbra 5MAX (labeled-ID $=0.054 \mathrm{in}$ ), ACE 68 (labeled-ID = 0.068 in) and JET 7 (labeled-ID = 0.072 in) (Penumbra, Inc, CA, USA), Microvention Sofia (labeled-ID $=0.055 \mathrm{in}$ ) and Sofia Plus (labeled-ID $=0.070 \mathrm{in}$ ) (Microvention, CA, USA), Stryker AXS Cat 6 (labeled-ID $=0.060$ in), and Cat 7 (labeled-ID $=0.068$ in) (Stryker, MI, USA), Medtronic React 68 (labeled-ID $=0.068 \mathrm{in}$ ) and React 71 (labeled-ID $=0.071 \mathrm{in}$ ) (Medtronic, MN, USA).

\section{Experimental setup}

A custom-made methacrylate deposit constituted the experimental setup with a connector at the bottom part, a 3D printed carotid artery model, a silicone tube simulating the femoral artery attached to a hemostatic valve, and a tubing system that connects all mentioned components. Figure 1 depicts the setup.

Regarding the in vitro carotid artery model, we selected a set of computed tomography angiography (CTA) images from an anonymized stroke patient. We then performed medical segmentation to get the 3D mesh of the common carotid artery, internal carotid artery (ICA), and proximal ramifications of the external carotid artery. This preliminary model was further processed to be printable, and it was manufactured by stereolithography, a 3D-printing technique that uses photoreactive resin and 
UV light to create the desired geometry. Softwares utilized in the manufacturing process were 3D Slicer [13], [14], Autodesk Meshmixer [15], and PreForm (Formlabs, Inc, MA, USA). The carotid artery was printed with a commercially available Elastic 50A resin by a high-resolution desktop 3D Printer, Form 3 (Formlabs, Inc, MA, USA).

The stiff clot analog was likewise 3D printed with the Elastic 50A resin; it was created with a conical geometry of $20 \mathrm{~mm}$ length $\times 5 \mathrm{~mm}$ diameter at the base (distal end) that progressively reduces at 4:1 length-diameter ratio until reaching the tip (proximal end). In all cases, the clot analog was partially introduced into the catheter, simulating the scenario where a thrombus cannot be completely aspirated but needs to be engaged by negative pressure and retrieved into the guiding catheter.

\section{Aspiration force study}

Concerning the experimental preparation, the experimental setup was filled with water, and the carotid artery model was coupled to the connector in the deposit. The clot analog's distal end was attached to the load cell using a suture thread, and the entire clot was inserted into the terminal ICA, partially occluding the vessel.

Asp-F were measured with a Zwick-Roell Zwick-line Z0.5TN universal testing machine (ZwickRoell, Ulm, Germany) equipped with $5 \mathrm{~N}$ load cell operating in traction mode controlled by testXpert II software (Zwick Roell Group Ltd., UIm, DE). We introduced the selected catheter through the hemostatic valve for each measurement and navigated it to reach the proximal end of the clot in the ICA. As a vacuum generator, we used a 60-cc syringe connected to the catheter's proximal end through a three-way valve. Once that aspiration started, the thrombus was engaged into the catheter, and the load cell started to shift at $1 \mathrm{~mm} / \mathrm{s}$ in the opposite direction to aspiration. Simultaneously, the device's force on the clot to resist the detachment was monitored to determine the peak value of asp-F. Each set of experiments per catheter was replicated 20 times $(N=20)$.

\section{Catheter tip mechanical behavior analysis}

Catheter tips' mechanical behaviors were characterized by the mechanical testing machine operating in compression mode. Figure $2 \mathrm{~A}$ depicts the experimental setup. Briefly, the catheter was subjected to a gripper $3 \mathrm{~mm}$ below the tip, the distal end of the conical clot was glued to the upper plate of the Zwick Roell machine, and its tip was positioned concentrically to the catheter tip.

Compression mode tests were configured as follows (figure 2B): the initial height position (h0) of the clot analog was adjusted, so its diameter matched the labeled-ID of each catheter. For this purpose, we shift the upper plate towards the device until the load cell detected the minimum changes in force, which meant that the clot was in contact with the catheter's inner walls; once a test started, the upper plate descending velocity towards the catheter was set to $0.25 \mathrm{~mm} / \mathrm{s}$, and the measurement was configured to finalize once the compression force matched the peak value determined in the asp-F study for each specific catheter. The testXpert II software registered the distance traveled by the upper plate from the initial height position, which corresponded to the clot's additional length inserted into the catheter tip. Hence, the effective ID (eff-ID) was defined as the catheter's ID when maximum asp-F was applied (equation 1):

$$
\text { effID }=D_{\text {clot }(h 0)}+\Delta L \frac{D}{L_{T}}
$$


Where $D_{\text {clot(ho) }}$ was equal to labeled-ID of each device, $\Delta L$ is the distance travelled until achieving the peak asp-F of each catheter, and $\frac{D}{L_{T}}=\frac{5 \mathrm{~mm}}{20 \mathrm{~mm}}$ is the conical clot's base diameter by length. The eff-ID allowed us to calculate the percentage of catheter tip widening $(\Delta I D)$ as a parameter to quantify devices' distensibility.

\section{Statistics}

Experimental asp-F were presented as mean \pm standard deviation. T-student test with a confidence interval of $95 \%$ was used to analyze asp-F delivered by catheters with the same labeled-ID, namely, ACE 68, Cat 7, and React 68.

Under the simulated scenario where there was no backflow through the catheter because of the occluded tip, the theoretical asp- $\mathrm{F}$ transmitted through a catheter is defined by equation 2 .

$$
a s p F=P \times A
$$

Where $P$, which has a constant value of $89.74 \pm 0.34 \mathrm{kPa}$, is the vacuum pressure generated by a $60 \mathrm{cc}$ syringe [10], and $A$ is the section area of different catheter tips. We calculated the relative difference between the experimental and theoretical asp-F ( $\Delta$ Force) and conducted a bivariate correlation to find the Pearson's correlation coefficient between $\Delta$ Force and $\Delta \mathrm{ID}$ measured from catheter tip distensibility study. Correlations between catheter's asp-F, labeled-ID and eff-ID were determined likewise using SPSS Statistics (IBM Corp., NY, USA).

\section{RESULTS}

Experimental asp- $\mathrm{F}$ of thrombectomy catheters in the carotid model under flow arrest scenario is shown in Figure 3A. The observed experimental asp-F ranged from 210 to $470 \mathrm{mN}$ (table 1). The correlation between the experimental asp-F and the catheter labeled-ID was confirmed by a Pearson's coefficient of 0.9601 ( $p<0.01$ ). Amongst all devices, React 71 delivered the highest asp- $F$ $(455.43 \pm 9.76 \mathrm{mN})$. Amongst catheters with the same lab ID (0.068 inches), experimental asp-F generated by Cat $7(382.23 \pm 5.26 \mathrm{mN})$ was higher than the experimental asp- $\mathrm{F}$ generated by React 68 (vs. $373.65 \pm 14.18 \mathrm{mN} ; p<0.05$ ). Both catheters showed higher experimental asp-F than ACE $68(338.10 \pm 5.69 \mathrm{mN} ; p<0.001)$

Theoretical asp- $F$ calculated from the product of vacuum pressure and the section area of the catheter tips are shown in Table 1 and plotted in Figure $3 \mathrm{~A}$. $\Delta$ Force was defined as the relative difference between the experimental asp-F and the theoretical asp- $\mathrm{F}$ for a given labeled-ID. The device that showed the minimum $\triangle$ Force was ACE 68 (60.80 \%), followed by Sofia $5 F(62.89 \%)$ and $5 \operatorname{MAX}(64.88 \%)$. React $71(98.68 \%)$ showed the highest $\triangle$ Force.

The catheter tip distensibility, observed when a stiff clot analog is aspirated, ranged between $14.69 \%$ (Jet 7 ) and $24.76 \%$ (React 71 ). Figure 3B shows $\Delta$ Force versus $\Delta I D$. Both variables showed a Pearson's correlation coefficient of 0.9050 ( $p<0.01)$, suggesting that catheter tip distensibility explains the discrepancy between measured (experimental) and expected (theoretical) asp$F$. Figure $3 \mathrm{C}$ presents experimental asp- $\mathrm{F}$ versus eff-ID. Both variables are correlated with a Pearson's coefficient of $0.9944(p<0.01)$.

\section{DISCUSSION}

In this study, we simulated an ADAPT scenario where a stiff clot larger than catheter ID cannot be directly aspirated and instead has to be engaged into the catheter tip for retrieval. Our results showed that overall labeled-ID correlated with the achieved experimental asp-F, however, we observed that larger catheters did not necessarily lead to the highest asp-F, or catheters with identical labeled-ID showed up to $13.05 \%$ variability in their experimental asp-F. Our findings, in line with previous publications [10], confirm that labeled-ID is not the only factor determining the experimental asp- $\mathrm{F}$ of a device and therefore, additional variables should be considered.

Long et al. [16] presented a novel catheter design and demonstrated that a larger proximal ID could [17] also increase the eff-ID of the catheter and consequently, the asp-F. Boisseau et al. [12] re- 
ported in their review that, besides catheter labeled-ID, other factors like the use of cyclical aspiration [18], [19] or balloon guide catheter [17], the contact angle between clot and catheter [20] and the clot composition [21] may influence the success of aspiration.

Several studies have reported that ADAPT may be more effective for fibrin-rich clots than for red blood cell-rich ones [12], [21], which suggests that thrombus-catheter tip biomechanical interaction has a significant influence on the recanalization rate. Our experiments identified that the catheter tip distensibility plays a significant role when applying the ADAPT technique to a stiff clot, generally both fibrin-rich and calcified types [22]. The tip distensibility may vary depending on materials used to manufacture the outer layer of the catheter; whereas React 68's outer jacket materials are based on a mix of polyamide and polyether, ACE 68 and Cat 7 add polyurethane to that mix, and, therefore, increasing the tip's resistance to strain and possibly the overall structural support."

By quantifying the distensibility of the tip, we were able to differentiate the catheter labeled-ID from the eff-ID, which is an experimental variable that also takes into account the mechanics of clotcatheter tip interaction. As aspiration devices may present a substantial tip distensibility, eff-ID should be considered to estimate the device efficiency rather than the labeled-ID specified by the manufacturer. Thus, our findings can be insightful for device selection criteria if we could identify thrombus' stiffnesses before the interventional procedure.

With a closer glance at Figure $3 \mathrm{~B}$, we observe that the correlation coefficient between $\Delta$ Force and $\Delta I D$ was excellent $(r=0.905 ; p<0.01)$ meaning that the difference between the expected and observed forces is mainly explained by the tip's distensibility. After adjusting the data for tip distensibility, only $0.56 \%$ of the $\Delta$ Force remains unexplained (figure $3 \mathrm{C}$ ). Therefore, at least in our experimental model, the impact of other features on $\Delta$ Force, such as the clot-catheter inner wall friction (that could also increase asp-F) is residual.

Several studies evaluated commercially available pumps: Froehler et al. [9] reported that Penumbra Max, ASPIRE device, and 60 cc syringe generate similar vacuum pressures (26-28 inHg); Yaeger et al. [10] conducted a technical comparison between Penumbra Jet Engine, Penumbra Max, Microvention Gomco, Stryker Medela, and $60 \mathrm{cc}$ syringe. They concluded that Penumbra Jet Engine generated the highest vacuum pressure $(28.8 \pm 0.10 \mathrm{inHg})$, although the others were close (26-27 inHg); and Gross et al. [23] validated in an in vitro model and clinically that aspiration with a $60 \mathrm{cc}$ syringe is as effective as Penumbra Max. Thus, results obtained in our tip force measurements using a manual aspiration syringe might be valid if commercially available pumps with comparable vacuum pressures are applied.

\section{Limitations}

The main limitation of this work is the clot mimic's stiffness, which may significantly differ from thrombus retrieved from stroke patients. Therefore, we aim to synthesize clot analogs that better reproduce physiological clot biomechanics in the following stages of our device evaluation studies. Furthermore, the influence of other variables such as catheter-vessel ratio and the concomitant presence of a stent retriever will be analyzed in future works.

\section{CONCLUSIONS}

When retrieving stiff thrombi with ADAPT, the difference between the theoretical asp-F according to labeled-ID and the measured asp-F is mainly explained by their tip's mechanical flexible behavior. Our results may help in the optimization of thrombectomy strategies and the development of novel aspiration catheters.

\section{Funding}

The authors thank the European Commission-ERANET (nAngioderm JTC2018-103) and the Spanish Ministry MICINN for the funding support (MAT2015-62725-ERC, RTI2018-096320-B-C21, and 
RTI2018-097038-B-C22,), the Severo Ochoa Program for Centers of Excellence and R\&D 20162019 and Obra Social la Caixa (Caixalmpulse Cl0015). The authors also thank the CERCA Program, and by the Commission for Universities and Research of the Department of Innovation, Universities, and Enterprise of the Generalitat de Catalunya (SGR2017-359), CIBER-BBN and the Spanish network of cell therapy (TERCEL).

\section{Competing interests}

The authors declare no competing interests.

\section{Contributorship}

$\mathrm{JL}, \mathrm{OC}$, MR conceived the design, performed the experiments, analyzed the data, and drafted the manuscript. AT, MDL, PC and EE made contributions to data interpretation and critical review for intellectual content.

\section{Data sharing}

All data are available upon reasonable request to the corresponding author. 


\section{REFERENCES}

[1] J. L. Saver et al., "Time to treatment with endovascular thrombectomy and outcomes from ischemic stroke: Ameta-analysis," JAMA - J. Am. Med. Assoc., vol. 316, no. 12, pp. 12791288, 2016, doi: 10.1001/jama.2016.13647.

[2] M. Goyal et al., "Endovascular thrombectomy after large-vessel ischaemic stroke: A metaanalysis of individual patient data from five randomised trials," Lancet, vol. 387, no. 10029, pp. 1723-1731, 2016, doi: 10.1016/S0140-6736(16)00163-X.

[3] O. O. Zaidat et al., "First pass effect: A new measure for stroke thrombectomy devices," Stroke, vol. 49, no. 3, pp. 660-666, 2018, doi: 10.1161/STROKEAHA.117.020315.

[4] A. M. Spiotta, K. M. Fargen, M. I. Chaudry, R. Turner, and A. S. Turk, "ADAPT: A Direct Aspiration First Pass Technique," Stroke, vol. 15, no. 2, pp. 68-70, 2016.

[5] F. Lally et al., "In vitro experiments of cerebral blood flow during aspiration thrombectomy: Potential effects on cerebral perfusion pressure and collateral flow," J. Neurointerv. Surg., vol. 8, no. 9, pp. 969-972, 2016, doi: 10.1136/neurintsurg-2015-011909.

[6] Y. Shi, D. Cheshire, F. Lally, and C. Roffe, "Suction force-suction distance relation during aspiration thrombectomy for ischemic stroke: A computational fluid dynamics study," Phys. Med., vol. 3, pp. 1-8, 2017, doi: 10.1016/j.phmed.2016.11.001.

[7] O. Nikoubashman, D. Wischer, H. M. Hennemann, M. Büsen, C. Brockmann, and M. Wiesmann, "Under pressure: Comparison of aspiration techniques for endovascular mechanical thrombectomy," Am. J. Neuroradiol., vol. 39, no. 5, pp. 905-909, 2018, doi: 10.3174/ajnr.A5605.

[8] Y. C. Hu and M. F. Stiefel, "Force and aspiration analysis of the ADAPT technique in acute ischemic stroke treatment," J. Neurointerv. Surg., vol. 8, no. 3, pp. 244-246, 2016, doi: 10.1136/neurintsurg-2014-011563.

[9] M. T. Froehler, "Comparison of Vacuum Pressures and Forces Generated by Different Catheters and Pumps for Aspiration Thrombectomy in Acute Ischemic Stroke," Interv. Neurol., vol. 6, no. 3-4, pp. 199-206, 2017, doi: 10.1159/000475478.

[10] K. Yaeger et al., "A technical comparison of thrombectomy vacuum aspiration systems," J. Neurointerv. Surg., vol. 12, no. 1, pp. 72-76, 2020, doi: 10.1136/neurintsurg-2019014929 . 
[11] D. Fernandez-Sanchez et al., "Suction force rather than aspiration flow correlates with recanalization in hard clots: an in vitro study model," J. Neurointerv. Surg., p. neurintsurg2020-017242, 2021, doi: 10.1136/neurintsurg-2020-017242.

[12] W. Boisseau et al., "Direct aspiration stroke thrombectomy: A comprehensive review," J. Neurointerv. Surg., vol. 12, no. 11, pp. 1099-1106, 2020, doi: 10.1136/neurintsurg-2019015508.

[13] SlicerSolutions, "3D Slicer 4.10.2," 2019. https://www.slicer.org/.

[14] R. Kikinis, S. D. Pieper, and K. G. Vosburgh, "3D Slicer: A Platform for Subject-Specific Image Analysis, Visualization, and Clinical Support," in Intraoperative Imaging and ImageGuided Therapy, New York, NY: Springer New York, 2014, pp. 277-289.

[15] Autodesk, "Autodesk MeshMixer 3.5," 2018. https://www.meshmixer.com/.

[16] T. D. Long et al., "Novel aspiration catheter design for acute stroke thrombectomy," J. Neurointerv. Surg., vol. 11, no. 2, pp. 179-183, 2019, doi: 10.1136/neurintsurg-2017013702.

[17] D. H. Kang et al., "Effect of balloon guide catheter utilization on contact aspiration thrombectomy," J. Neurosurg., vol. 131, no. 5, pp. 1494-1500, 2019, doi: 10.3171/2018.6.JNS181045.

[18] S. Simon, C. P. Grey, T. Massenzo, D. G. Simpson, and P. W. Longest, "Exploring the efficacy of cyclic vs static aspiration in a cerebral thrombectomy model: An initial proof of concept study," J. Neurointerv. Surg., vol. 6, no. 9, pp. 677-683, 2014, doi: 10.1136/neurintsurg-2013-010941.

[19] R. A. Arslanian et al., "Complete clot ingestion with cyclical ADAPT increases first-pass recanalization and reduces distal embolization," J. Neurointerv. Surg., vol. 11, no. 9, pp. 931-936, 2019, doi: 10.1136/neurintsurg-2018-014625.

[20] G. Bernava et al., "Direct thromboaspiration efficacy for mechanical thrombectomy is related to the angle of interaction between the aspiration catheter and the clot," $\mathrm{J}$. Neurointerv. Surg., vol. 12, no. 4, pp. 396-400, 2020, doi: 10.1136/neurintsurg-2019015113.

[21] G. Ye, R. Cao, J. Lu, P. Qi, J. Chen, and D. Wang, "Association between thrombus density and reperfusion outcomes using different thrombectomy strategies: A singlecenter 
study and meta-analysis," Front. Neurol., vol. 10, no. JUL, 2019, doi:

10.3389/fneur.2019.00843.

[22] J. Y. Chueh, A. K. Wakhloo, G. H. Hendricks, C. F. Silva, J. P. Weaver, and M. J. Gounis, "Mechanical characterization of thromboemboli in acute ischemic stroke and laboratory embolus analogs," Am. J. Neuroradiol., vol. 32, no. 7, pp. 1237-1244, 2011, doi: 10.3174/ajnr.A2485.

[23] B. A. Gross, A. P. Jadhav, T. G. Jovin, and B. T. Jankowitz, "Dump the pump: Manual aspiration thrombectomy (MAT) with a syringe is technically effective, expeditious, and cost-efficient," J. Neurointerv. Surg., vol. 10, no. 4, pp. 354-357, 2018, doi: 10.1136/neurintsurg-2017-013520. 


\begin{tabular}{|c|c|c|c|c|c|c|c|c|}
\hline Catheter & $\begin{array}{l}\text { labeled-ID } \\
\text { (in) }\end{array}$ & \multicolumn{3}{|c|}{ Experimental } & $\begin{array}{l}\text { Theoretical } \\
\text { Asp-F }(\mathrm{mN})\end{array}$ & $\begin{array}{c}\Delta \text { Force } \\
(\%)\end{array}$ & $\begin{array}{l}\Delta \mathrm{ID} \\
(\%)\end{array}$ & $\begin{array}{l}\text { eff-ID } \\
\text { (in) }\end{array}$ \\
\hline 5 MAX & 0.054 & 218.62 & \pm & 4.66 & 132.60 & 64.88 & 17.93 & 0.064 \\
\hline Sofia 5F & 0.055 & 224.06 & \pm & 7.53 & 137.55 & 62.89 & 16.32 & 0.064 \\
\hline Cat 6 & 0.060 & 286.65 & \pm & 5.28 & 163.70 & 75.11 & 17.95 & 0.071 \\
\hline React 68 & 0.068 & 373.65 & \pm & 14.18 & 210.26 & 77.71 & 19.12 & 0.081 \\
\hline ACE 68 & 0.068 & 338.10 & \pm & 5.69 & 210.26 & 60.80 & 16.08 & 0.079 \\
\hline Cat 7 & 0.068 & 382.23 & \pm & 5.26 & 210.26 & 81.79 & 18.83 & 0.081 \\
\hline Sofia Plus & 0.070 & 392.21 & \pm & 12.27 & 222.81 & 76.03 & 18.53 & 0.083 \\
\hline React 71 & 0.071 & 455.43 & \pm & 9.67 & 229.22 & 98.68 & 24.76 & 0.089 \\
\hline Jet 7 & 0.072 & 396.75 & \pm & 9.49 & 235.72 & 68.31 & 14.69 & 0.083 \\
\hline
\end{tabular}

Table 1. Aspiration force (Asp-F) and catheter tip mechanical behavior analysis. LabID is the catheter inner diameter specified by device manufacturers, theoretical Asp-F was calculated by applying eq. $2, \Delta$ Force is the relative difference between experimental and theoretical Asp-F, $\Delta \mathrm{ID}$ obtained from eq. 1 is a parameter that quantifies catheter tip's distensibility and eff-ID is the catheter ID considering thrombus-device mechanical interaction.

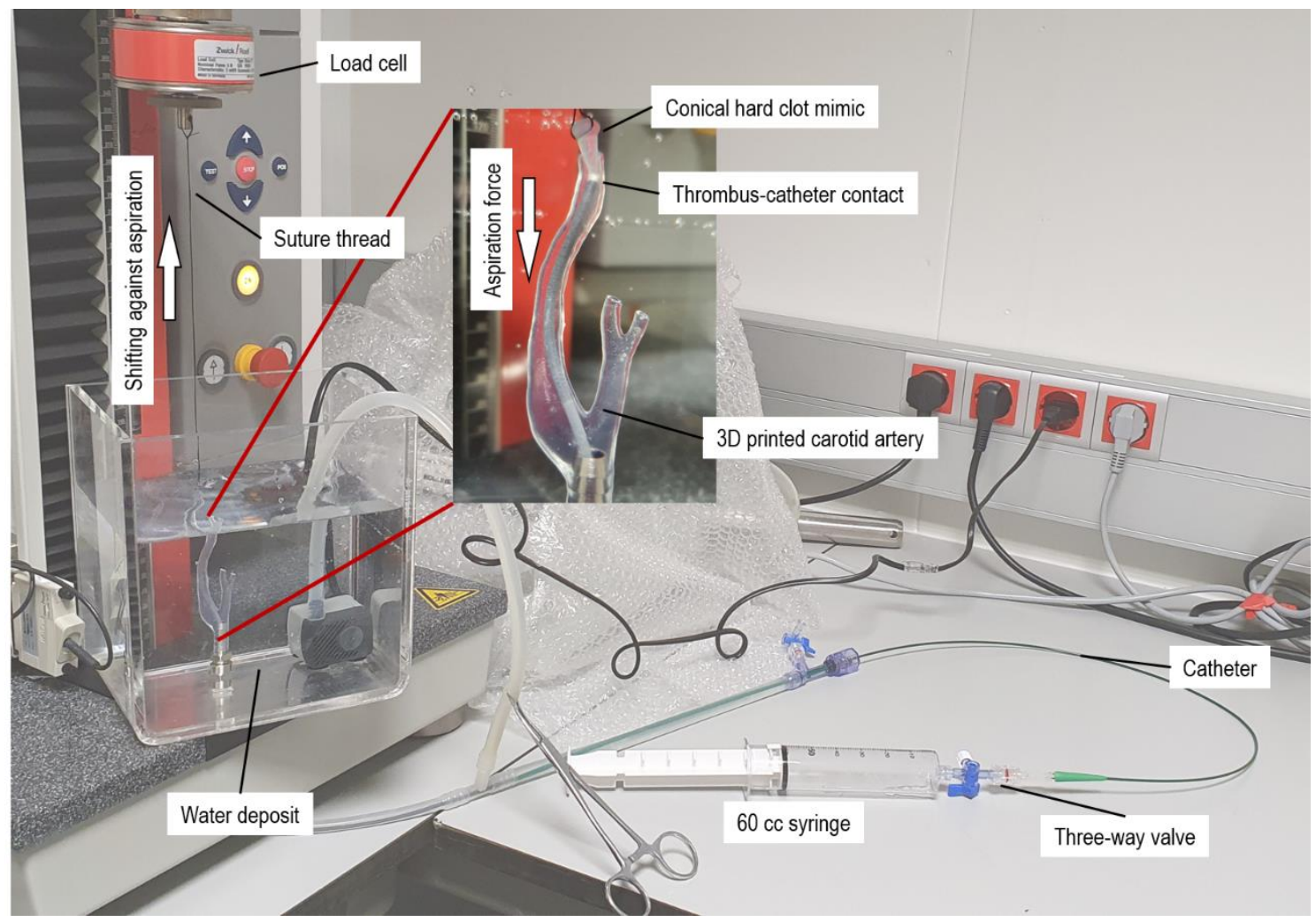

Figure 1. Experimental setup to measure aspiration forces exerted by thrombectomy catheters on a stiff clot mimic. 


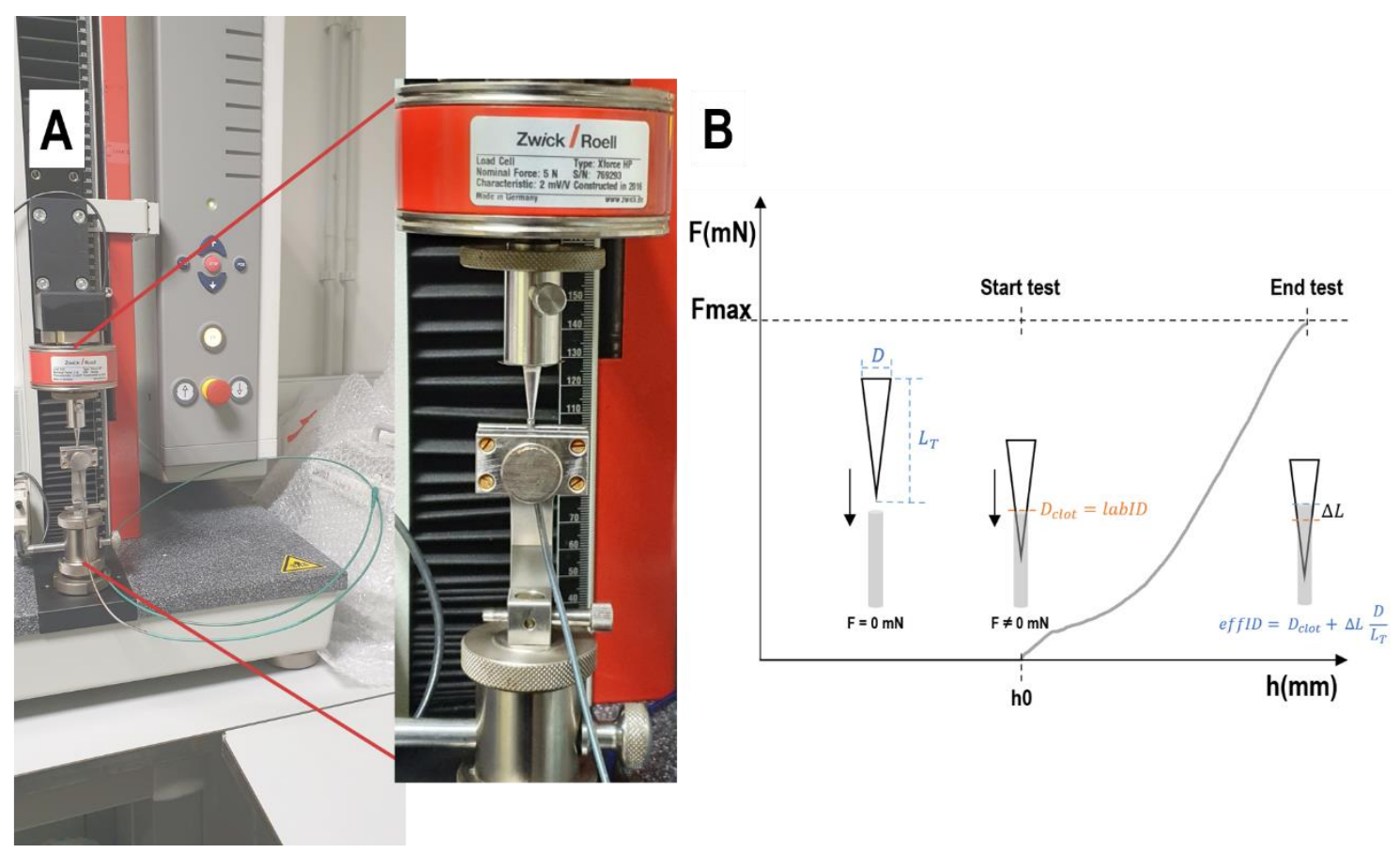

Figure 2. A) Experimental setup to determine catheters tip distensibility, B) force (F) vs. height position (h) concept plot, and the distinction between different stages of compression test to find the effID. $D$ and $L_{T}$ are the base diameter and the total length of the conical thrombus, $D_{\text {clot }}$ is the clot mimic diameter at the beginning of the test, $\Delta \mathrm{L}$ is the additional length inserted into the device and Fmax is the experimental aspF determined for each catheter. 

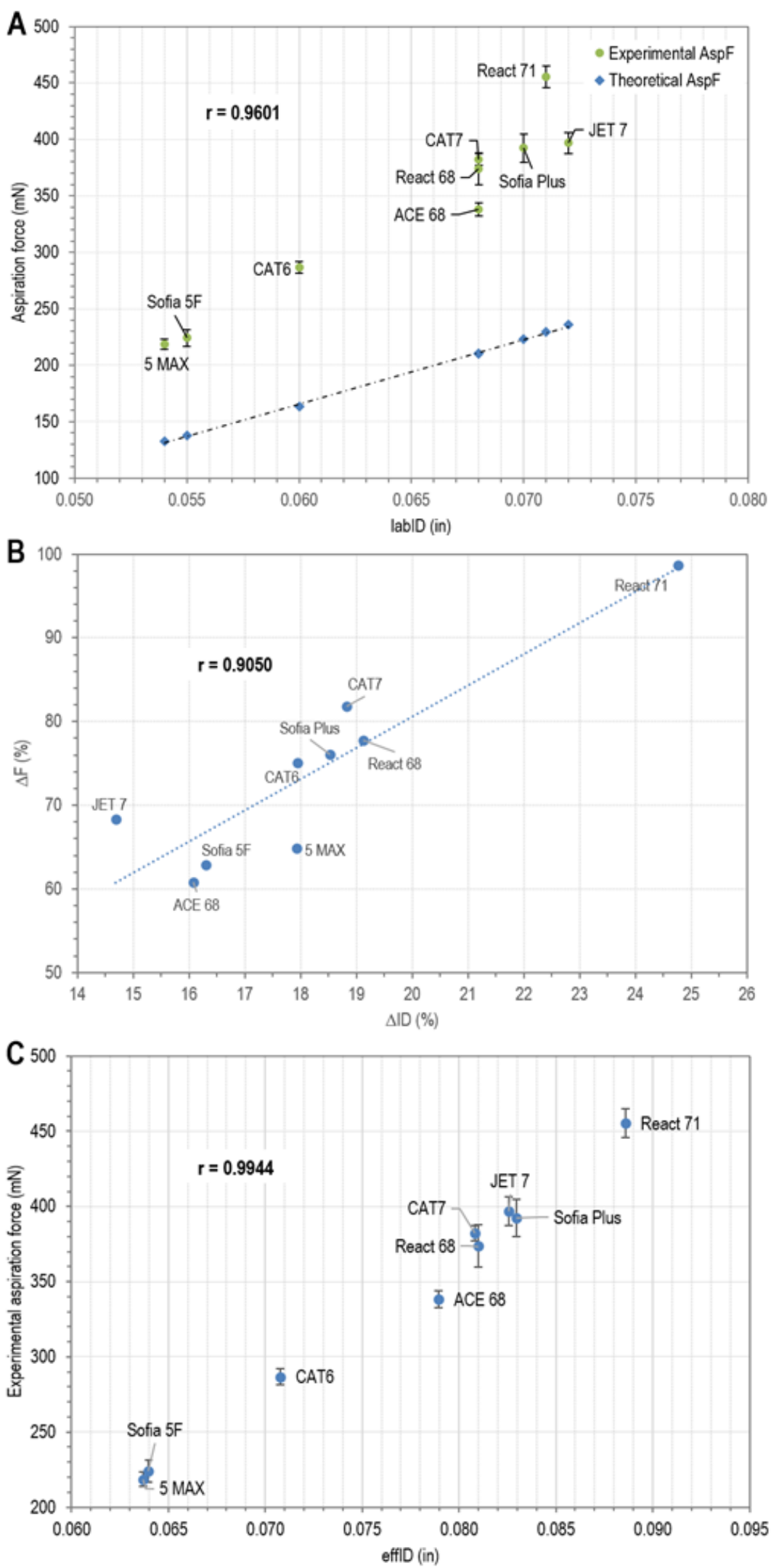

Figure 3. A) Experimental vs. theoretical AspF; B) the relative difference between measured and expected AspF is correlated to catheter tip distensibility; C) effID is a better parameter to estimate AspF than ID specified by manufacturers. 